\title{
Editorial: The 20th Anniversary of the Extremes Journal
}

\author{
Holger Rootzén ${ }^{1}$ • Jürg Hüsler² • Thomas Mikosch ${ }^{3}$ \\ Published online: 4 September 2018 \\ (C) Springer Science+Business Media, LLC, part of Springer Nature 2018
}

This year we celebrate the 20th anniversary of the founding of our journal.

In 1997 Holger Rootzén (Chalmers University Gothenburg) was asked by John Martindale from the publisher Kluwer to be the first editor of a new journal, to be called Extremes. The goal of the journal was to publish scientific papers on rigorous probabilistic and statistical modeling and serious data analyses for extreme random objects.

The first issue of the journal Extremes was published in 1998, 20 years ago. It contained 4 papers, on the probability of failure of a sea-wall; on diffusion models in finance; on heavy-tailed sums; and on scan statistics. Since then, these papers have been cited 530 times in total. Papers in Extremes have explored all parts of the stochastic theory of extreme values and extreme order statistics, which were used to study the strength of materials, extreme floods and storms, large ocean waves, ship safety, and large claims in insurance. Extremes in finance have been a recurring topic. Recently, increasing risks of dangerous environmental extremes caused by climate change have become an important inspiration and focus.

Certainly not by accident, 1998 was also the year when the First International Conference on Extreme Value Analysis (EVA) was held, at Chalmers University Gothenburg, Holger Rootzén's home institution. Since then, the journal Extremes and the biannual International EVA Conferences have co-existed in a natural symbiosis. The journal has published papers previously presented at EVA conferences such as state-of-the-art surveys on software for the statistics of extremes, discussion papers on the use of copulas or the longest human lifespan, and a data challenge on the prediction of extreme precipitation.

Thomas Mikosch

mikosch@math.ku.dk

1 Chalmers University of Technology, Göteborg, Sweden

2 University of Bern, Bern, Switzerland

3 University of Copenhagen, Copenhagen, Denmark 
Since 2004 Springer Publishing (now Springer Nature) published the Extremes Journal because Springer bought Kluwer. As a consequence, Extremes was included in the Springer bundle of mathematics and statistics journals, and the financial situation of the journal stabilized. Since 2007 the new editor-in-chief Jürg Hüsler was able to attract an increasing number of submissions and to guarantee an improvement of the quality of the published papers. As a result, Extremes also became more visible in probability/statistics circles and related areas.

In 2015 Thomas Mikosch became the new editor-in-chief of Extremes. During his term as editor the journal has received 120 submissions per year, and more than 30 high quality papers could be published every year.

Extreme value theory is an integral part of general probability theory and statistics. Its importance continues to grow, both in other parts of probability theory and for a broad range of important practical problems. The topics of the submitted papers reflect the main stream in these fields. Next to classical problems of extreme value analysis, such as estimation and fitting of extreme value models, high quantile and tail estimation, and the study of patterns of exceedances of stochastic processes above high levels, new topics have entered the field. Among them are the strong field of modeling, simulation and estimation of max-stable random fields, the world of multivariate extremes, extremes for time series, extreme eigenvalues of large random matrices, .... The cosmos of extremes will surely benefit from new advances in machine learning, statistics of high-dimensional data, statistics of social networks, random graph and branching theory. Conversely, extreme value theory provides useful methods to these areas. The recent EVA conferences have shown that the extreme value community is in touch with and part of these new developments.

A successful journal depends on the efficiency and competence of its editorial board and its reviewers. The journal Extremes has been in the lucky situation that our Associate Editors and reviewers are top experts. This fact has allowed the editors to conduct fast decision processes on the submitted papers.

We (the past and present editors of the journal) would like to take the opportunity and thank the Associate Editors, the reviewers, the authors, and the readers of Extremes for their constant support. We are looking into a bright future of the Extremes Journal.

Holger Rootzén

September 1, 2018

Jürg Hüsler

Thomas Mikosch 